PROCEEDINGS OF THE

AMERICAN MATHEMATICAL SOCIETY

Volume 136, Number 8, August 2008, Pages 2741-2744

S 0002-9939(08)09283-6

Article electronically published on April 3, 2008

\title{
A CRITERION FOR GREENBERG'S CONJECTURE
}

\author{
LUCA CAPUTO AND FILIPPO ALBERTO EDOARDO NUCCIO \\ (Communicated by Ken Ono)
}

\begin{abstract}
We give a criterion for the vanishing of the Iwasawa $\lambda$-invariants of totally real number fields $K$ based on the class number of $K\left(\zeta_{p}\right)$ by evaluating the $p$-adic $L$-functions at $s=-1$.
\end{abstract}

\section{INTRODUCTION}

Let $K$ be a real abelian number field and let $p$ be an odd prime. Set $F=K\left(\zeta_{p}\right)$ where $\zeta_{p}$ is a primitive $p$-th root of unity and $H=\operatorname{Gal}(F / K)$. Set, moreover, $G=\operatorname{Gal}(F / \mathbb{Q})$ and $\varpi=\operatorname{Gal}\left(\mathbb{Q}\left(\zeta_{p}\right) / \mathbb{Q}\right)$. So the diagram of our extensions is as follows:

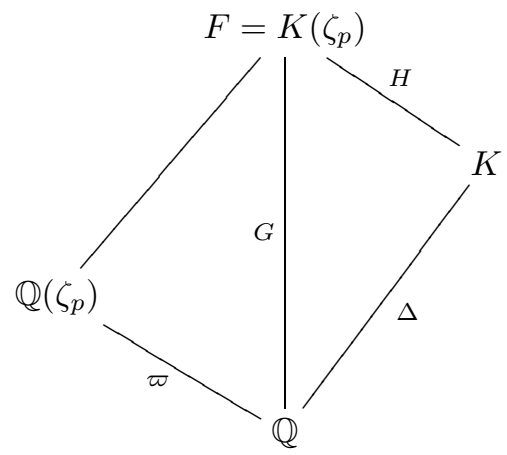

Let $\tilde{\omega}: H \rightarrow \mathbb{Z}_{p}^{\times}$and $\omega: \varpi \rightarrow \mathbb{Z}_{p}^{\times}$be the Teichmüller characters of $K$ and $\mathbb{Q}$, respectively. We give (Theorem 2.3) a criterion under which a set of odd Iwasawa invariants associated with $F$ vanishes: by means of a Spiegelungsatz, these odd invariants make their even mirrors vanish too. In the case $p=3$ (Corollary 2.5) or $p=5$ and $[K: \mathbb{Q}]=2$ (Theorem 2.7) this allows us to verify a conjecture of Greenberg for the fields satisfying our criterion.

\section{MAin RESUlt}

Proposition 2.1. The following equality holds:

$$
r k_{p}\left(\mathrm{~K}_{2}\left(\mathcal{O}_{K}\right)\right)=r k_{p}\left(\left(C l_{F}^{\prime}\right)_{\omega^{-1}}\right)+|S|,
$$

Received by the editors June 8, 2007, and, in revised form, July 2, 2007.

2000 Mathematics Subject Classification. Primary 11R23; Secondary 11R70.

Key words and phrases. Iwasawa theory, Greenberg's Conjecture, $K$-theory of Global Fields.

The second author wishes to thank the "Laboratoire de Mathématiques Nicolas Oresme" de Caen for its kind hospitality during the period in which this work has been done.

(C)2008 American Mathematical Society Reverts to public domain 28 years from publication 
where $\mathrm{K}_{2}\left(\mathcal{O}_{K}\right)$ is the tame kernel of $K$-theory, $C l_{F}^{\prime}$ is the class group of the ring $\mathcal{O}_{F}[1 / p]$ (and we take its $\tilde{\omega}^{-1}$-component for the action of $H$ ) and $S$ is the set of p-adic primes of $K$ which split completely in $F$.

Proof. This result dates back to Tate: for an explicit reference, see [Gra, Theorem 7.7.3.1.

Proposition 2.2. Suppose that $\mathbb{Q}\left(\zeta_{p}\right)$ is linearly disjoint from $K$ over $\mathbb{Q}$. Then the following equalities hold:

$$
\begin{aligned}
& v_{p}\left(\left|\mathrm{~K}_{2}\left(\mathcal{O}_{K}\right)\right|\right)=v_{p}\left(\zeta_{K}(-1)\right) \quad \text { if } p \geq 5, \\
& v_{3}\left(\left|\mathrm{~K}_{2}\left(\mathcal{O}_{K}\right)\right|\right)=v_{3}\left(\zeta_{K}(-1)\right)+1,
\end{aligned}
$$

where $v_{p}$ denotes the standard $p$-adic valuation and $\zeta_{K}$ is the Dedekind zeta function for $K$.

Proof. The Birch-Tate conjecture, which has been proved by Mazur, Wiles and by Greither (since it is a consequence of the Main Conjecture in Iwasawa theory), tells us that

where

$$
\frac{\left|K_{2}\left(\mathcal{O}_{K}\right)\right|}{w_{2}}=\zeta_{K}(-1)
$$

$$
w_{2}=\max \left\{n \in \mathbb{N} \mid \text { the exponent of } \operatorname{Gal}\left(K\left(\zeta_{n}\right) / K\right) \text { is } 2\right\} .
$$

By our hypothesis, $\mathbb{Q}\left(\zeta_{p}\right)$ is linearly disjoint from $K$ over $\mathbb{Q}$. Hence $F / K$ is Galois with cyclic Galois group of order $p-1$. If $p=3$, then for the same argument, $3 \mid w_{2}$ but $9 \nmid w_{2}$ since $K\left(\zeta_{9}\right) / K$ has degree 6 . Taking $p$-adic valuation we get the claim.

Theorem 2.3. Let $p \geq 5$. Suppose that the following hold:

- $K$ and $\mathbb{Q}\left(\zeta_{p}\right)$ are linearly disjoint over $\mathbb{Q}$;

- the set $S$ of Proposition 2.1 is empty;

- the Main Conjecture of Iwasawa theory holds for F.

Then, if $p$ does not divide the order of $C l_{F}\left(\tilde{\omega}^{-1}\right), \lambda_{\chi \omega^{2}}(F)=0$ for all characters $\chi$ of $\Delta$.

Proof. First of all, we should just prove the theorem for nontrivial characters of $\Delta$, since $\lambda_{\omega^{2}}=0$ as it corresponds to the $\omega^{2}$-part of the cyclotomic extension of $\mathbb{Q}\left(\zeta_{p}\right)$, which is always trivial: indeed, $B_{1 / 2}=-1 / 2$, and then Herbrand's theorem and Leopoldt's Spiegelungsatz (Was, Theorems 6.7 and 10.9) give $\lambda_{\omega^{2}}=0$.

By hypothesis, the set $S$ of Proposition (2.1) is empty. Therefore $r k_{p}\left(\mathrm{~K}_{2}\left(\mathcal{O}_{K}\right)\right)=$ 0 and Proposition (2.2) (which we can apply because $K$ verifies its hypothesis) together with $p \geq 5$ tells us that $v_{p}\left(\zeta_{K}(-1)\right)=0$. Since we can factor

$$
\zeta_{K}(s)=\prod_{(\chi \in \hat{\Delta})} L(s, \chi)=\zeta_{\mathbb{Q}}(s) \prod_{\chi \neq 1} L(s, \chi)
$$

we find that

$$
v_{p}\left(\zeta_{K}(-1)\right)=\sum_{\chi \neq 1} v_{p}(L(-1, \chi))=0 .
$$

The interpolation formula for the $p$-adic $L$-function (see [Was, Chapter 5 ) tells us that

$$
L_{p}(-1, \chi)=\left(1-\chi \omega^{-2}(p) p\right) L\left(-1, \chi \omega^{-2}\right)
$$


now we invoke the Main Conjecture as stated in ([Gre, page 452) to relate these $L$ functions with the characteristic polynomials of some submodules of the Iwasawa module $X_{\infty}(F)$. Observe that the hypothesis of linear disjointness tells us that $\hat{G} \cong \hat{\Delta} \times \hat{\varpi}$, so we can split

$$
X_{\infty}(F) \cong \bigoplus_{\chi \in \Delta} \bigoplus_{i=1}^{p-1} X_{\infty}(F)\left(\chi \omega^{i}\right)
$$

where $G$ acts on $X_{\infty}(F)\left(\chi \omega^{i}\right)$ as $g \cdot x=\left(\chi \omega^{i}\right)(g) x$ for all $g \in G$ and $x \in X$. Then the Main Conjecture for $F$ allows us to write $L_{p}\left(-1, \chi \omega^{i}\right)=f\left(-p /(1+p), \chi^{-1} \omega^{1-i}\right)$ for all even $2 \leq i \leq p-1$, where $f\left(T, \chi^{-1} \omega^{1-i}\right) \in \mathbb{Z}_{p}[T]$ is the characteristic polynomial of $X_{\infty}(F)\left(\chi^{-1} \omega^{1-i}\right)$ : thus $L_{p}\left(-1, \chi \omega^{i}\right)$ is $\mathbb{Z}_{p}$-integral. Applying this for $i=2$ and plugging it in (2.2) we find $v_{p}(L(-1, \chi)) \geq 0$ for all $\chi$, and thanks to (2.1) we indeed find $v_{p}(L(-1, \chi))=0$ for all $\chi \in \hat{\Delta}$, so

$$
v_{p}\left(L_{p}\left(-1, \chi \omega^{2}\right)\right)=0 \quad \forall \chi \in \hat{\Delta} .
$$

If we now apply again the Main Conjecture, we find that this corresponds to

$$
v_{p}\left(f\left(\frac{1}{1+p}-1, \chi^{-1} \omega^{-1}\right)\right)=v_{p}\left(f\left(\frac{-p}{1+p}, \chi^{-1} \omega^{-1}\right)\right)=0 \quad \forall \chi \in \hat{\Delta} .
$$

Since $f\left(T, \chi^{-1} \omega^{-1}\right) \in \mathbb{Z}_{p}[T]$ is distinguished (see Was, Chapter 7 ) this is possible if and only if $\operatorname{deg}_{T}\left(f\left(T, \chi^{-1} \omega^{-1}\right)\right)=0$; but this is precisely the Iwasawa invariant $\lambda_{\chi^{-1} \omega^{-1}}$, so we have

$$
\lambda_{\chi^{-1} \omega^{-1}}=0 \quad \forall \chi \in \hat{\Delta} .
$$

Since the inequality $\lambda_{\chi^{-1} \omega^{-1}} \geq \lambda_{\chi \omega^{2}}$ is classical and well known (see, for instance, $[\mathrm{BN}]$, Section 4), we achieve the proof.

Remark 2.4. We should ask that the Main Conjecture holds for $K$ to apply it in the form of Gre. For this, it is enough that there exists a field $E$ that is unramified at $p$ and such that $F=E\left(\zeta_{p}\right)$, as is often the case in the applications. Moreover, we remark that the hypotheses of the theorem are trivially fulfilled if $p$ is unramified in $K / \mathbb{Q}$.

Corollary 2.5. Assume $p=3$. If 3 does not divide the order of $C l_{F}\left(\tilde{\omega}^{-1}\right)$ and it is unramified in $K$, then $\lambda(K)=\lambda(F)=0$.

Proof. First of all, the theorem applies for $p=3$ also, since we still have (2.1) thanks to $\zeta(-1)=-1 / 12$; moreover, $K$ is clearly disjoint from $\mathbb{Q}(\sqrt{-3})=\mathbb{Q}\left(\zeta_{3}\right)$, as it is unramified, and $F / K$ is ramified, so $S=\emptyset$. But in this case we have $\omega^{2}=1$, so the statement of the theorem is that all Iwasawa invariants $\lambda_{\chi}$ vanish for $\chi \in \hat{\Delta}$ and their sum is precisely $\lambda(K)$. Concerning $\lambda(F)$, in the proof of the theorem we first prove that all $\lambda_{\chi \omega}$ vanish, and deduce from it the vanishing of their "mirror" parts.

Remark 2.6. In the case that $K=\mathbb{Q}(\sqrt{d})$ is real quadratic, this is a classical result of Scholtz (although it is expressed in term of Iwasawa invariants); see Was], Theorem 10.10.

Theorem 2.7. Let $K$ be a real quadratic field and suppose that $5 \nmid\left|C l_{F}\right|$. Then $\lambda(K)=0$. 
Proof. Write $K=\mathbb{Q}(\sqrt{d})$ and let $\chi$ be its nontrivial character: the result being well known if $d=5$ we assume throughout that $d \neq 5$. Then we should consider two cases, namely $5 \mid d$ and $5 \nmid d$. We have the following diagram of fields (we don't draw the whole of it):

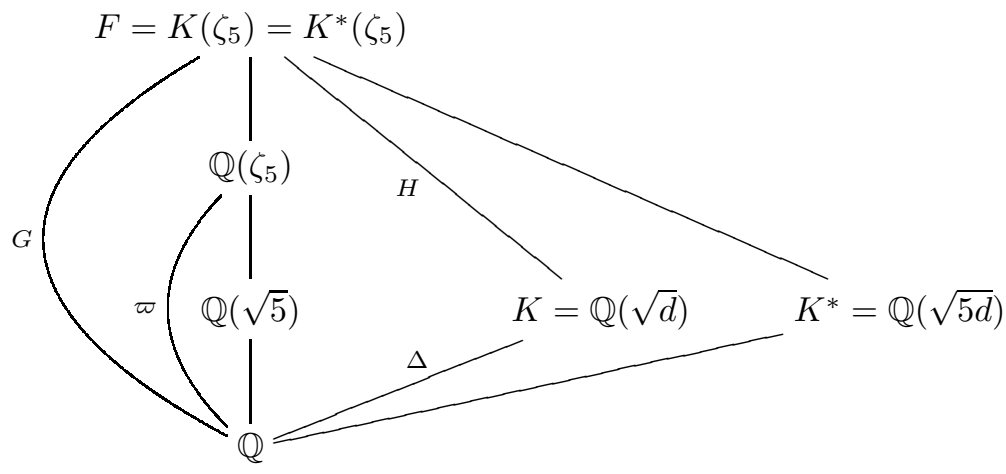

Suppose first of all that $5 \mid d$ or that 5 is inert in $K / \mathbb{Q}$. Since $5 \nmid[F: K]$, our hypothesis implies that $5 \nmid\left|C l_{K}\right|$ (see [Was, Lemma 16.15). But then we would trivially have $\lambda(K)=0$ as an easy application of Nakayama's Lemma (see Was, Proposition 13.22). We can thus suppose that 5 splits in $K / \mathbb{Q}$. We then apply Theorem 2.3 to $K^{*}$ instead of $K$ : since $\mathbb{Q}(\sqrt{5}) \subseteq \mathbb{Q}\left(\zeta_{5}\right)$, our field is linearly disjoint over $\mathbb{Q}$ from $\mathbb{Q}\left(\zeta_{5}\right)$ and $S=\emptyset$ thanks to degree computations. Moreover the Main Conjecture holds for $F$ since $F=K\left(\zeta_{5}\right)$ and $K$ is totally real and unramified at 5 . We find that $\lambda_{\omega^{2} \chi^{*}}=0$ where $\chi^{*}$ is the nontrivial character of $K^{*}$. But clearly $\chi^{*}=\chi \omega^{2}$, so $\lambda_{\chi}=0$. Since the Iwasawa invariant associated with the trivial character is $\lambda(\mathbb{Q})=0$, we have $\lambda(K)=\lambda(\mathbb{Q})+\lambda_{\chi}=0$.

\section{REFERENCES}

[BN] R. Badino And T. Nguyen Quang Do, Sur les égalités du miroir et certaines formes faibles de la conjecture de Greenberg, Manuscripta Mathematica, CXVI, 323-340 (2005). MR:2130946 (2006b:11133)

[Gra] G. Gras, Class field theory: From theory to practice, Springer Monographs in Mathematics, Springer-Verlag, 2003. MR1941965 (2003j:11138)

[Gre] C. Greither, Class groups of abelian fields, and the main conjecture, Annales de l'Institut Fourier, XLII, 449-499 (1992). MR1182638(93j:11071)

[Was] L. Washington, Introduction to Cyclotomic Fields, Graduate Texts in Mathematics, Vol. 83, Springer-Verlag, 1997. MR:1421575 (97h:11130)

Dipartimento di Matematica "Leonida Tonelli", Università degli studi di Pisa, Largo Bruno Pontecorvo, 5 - 56127 - Pisa, Italy

E-mail address: caputo@mail.dm.unipi.it

Dipartimento di Matematica "Guido Castelnuovo", Università degli studi "La Sapienza" Piazzale Aldo Moro, 5 - 00185 - Roma, Italy

E-mail address: nuccio@mat.uniroma1.it 